\title{
New faunistic records of Limoniidae and Tipulidae (Diptera) from the Czech Republic
}

\author{
Jaroslav Starý \& Alois Čelechovský
}

New faunistic records of Limoniidae and Tipulidae (Diptera) from the Czech Republic. - Acta Mus. Siles. Sci. Natur., 64: 111-113, 2015.

Abstract: Primary collecting data are given for 11 species of Limoniidae and Tipulidae, representing the first records from Bohemia or Moravia.

Key words: Diptera, Limoniidae, Tipulidae, new records, Czech Republic.

\section{Introduction}

In the present paper, findings of ten species of the family Limoniidae and one species of the Tipulidae are published, representing the first records from Bohemia or Moravia, the two main regions of the Czech Republic. The new records will be included in the third electronic version of the Checklist of Diptera of the Czech Republic and Slovakia, now in preparation.

\section{Material and methods}

Classification, nomenclature, and distribution for individual species are given as summarized by Oosterbroek (2015). Some specimens originally preserved in ethanol were dried and mounted on points. The male terminalia, if necessary, were prepared by boiling in a solution of $10 \% \mathrm{KOH}$ and preserved in glycerine in a sealed plastic tube pinned with the appropriate specimen.

\section{LIMONIIDAE}

\section{Ellipteroides (Protogonomyia) limbatus (von Roser, 1840)}

Bohemia bor.: Krkonoše Mts, Horní Lánov, Pekelský brook (5360), 550 m, Malaise trap, 30.6.-30.7.2009, 2 q, M. Barták leg., Horní Lánov, Bíner (5360), 609 m, Malaise trap, damp meadow (50³7'50.1"N 15²0'34.3"E), 16.6.-7.7.2009, 20̂, M. Barták \& J. Vaněk leg., all J. Starý det. et coll.

Distribution: Widely distributed in Europe, including Czech Republic (Moravia) and Slovakia; Turkey (Asiatic part). First records from Bohemia.

\section{Hoplolabis (Parilisia) yezoana (Alexander, 1924)}

Bohemia mer.: Malenice, Volyňka shores (6849), at light, 31.5.2004, 1ð J. J. Starý leg., det. et coll.

Distribution: Palaearctic species widely distributed in Europe, including Czech Republic (Moravia) and Slovakia, ranging as far east as Russian Far East and Japan. First record from Bohemia.

\section{Molophilus (Molophilus) priapoides Starý, 1971}

Bohemia bor.: Jizerské Mts, Janov nad Nisou, “Tichá říčka” (5257), 20.5.2014, 10 , J. Preisler leg., J. Starý det. et coll.

Distribution: Austria, Bulgaria, Czech Republic (Moravia), France, Germany, Italy, Poland, Romania, Serbia, Slovakia. First record from Bohemia. 


\section{Molophilus (Molophilus) undulatus Tonnoir in Goetghebuer \& Tonnoir, 1920}

Bohemia bor.: Krkonoše Mts, Horní Lánov, Pekelský brook (5360), 550 m, Malaise trap, 30.6.-30.7.2009, 1ð, 30.7.-18.8.2009, 1ð̄, Černý Důl, Zrcadlový brook (5360), 670 m, Malaise trap, 18.8.-1.9.2009, 1ô, all M. Barták leg., J. Starý det. et coll.

Distribution: Widely distributed in Europe, including Czech Republic (Moravia) and Slovakia. First records from Bohemia.

\section{Molophilus (Molophilus) variispinus Starý, 1971}

Bohemia bor.: Krkonoše Mts, Horní Lánov, Pekelský brook (5360), 550 m, Malaise trap, 5.2009, 10ิ, 30.6.30.7.2009, 1§, M. Barták leg., J. Starý det. et coll.

Distribution: Austria, Czech Republic (Moravia), Germany, Great Britain, Poland, Slovakia, Switzerland. First record from Bohemia.

\section{Ormosia (Ormosia) moravica Starý, 1969}

Bohemia bor.: Jizerské Mts, Raspenava, Oldřichovské sedlo (5156), Viničná cesta, 500 m, yellow pans, 17.5.3.6.2003, 10ิ J. Preisler \& P. Vonička leg.; Krkonoše Mts, Horní Lánov, Pekelský brook (5360), 550 m, Malaise

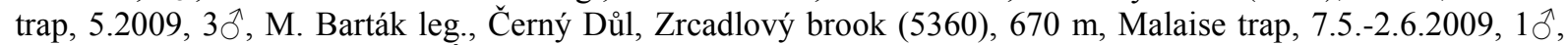
M. Barták leg., Svoboda nad Úpou, "Slunečná stráň" (5360), nr. pond, 645 m (50³8'12.5"N 1549'23.5"E), Malaise trap, 7.5.-2.6.2009, 4^ึ, J. Vaněk leg., all J. Starý det. et coll.

Distribution: Czech Republic (Moravia), Germany, Poland, Romania, Slovakia, Ukraine. First records from Bohemia.

\section{Scleroprocta sororcula (Zetterstedt, 1851)}

Bohemia bor.: Jizerské Mts, Oldřichov v Hájích, Špičák (5156), 600 m, yellow pans, 17.5.-3.6.2003, 1今̄, J. Preisler leg., Horní Maxov, “Malá Strana” (5257), 700-720 m, Malaise trap, 6.-16.5.2003, 2ð, 16.-29.5.2003 $1 \widehat{\jmath}$, J. Preisler \& P. Vonička leg., Jizerka, "Rybí loučky” peat-bog (5158), 850 m, Malaise trap, 17.-29.5.2003,

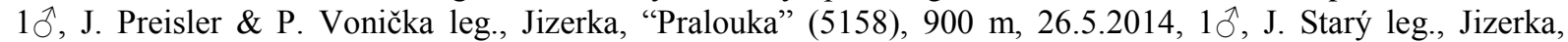
Bukovec (5158), 920 m, yellow pans, 5.-16.5.2003, 2ð, 16.-29.5.2003, 2ð, J. Preisler \& P. Vonička leg., Rejdice nr. Kořenov, Ješkrabec brook (5258), 650 m, emergence trap, 30.5.-16.6.2003, 1ठ, J. Preisler \& P. Vonička leg., all J. Starý det. et coll.

Distribution: Widely distributed in Europe except for the south, including Czech Republic (Moravia) and Slovakia. First records from Bohemia.

\section{Tasiocera (Dasymolophilus) exigua Savchenko, 1973}

Bohemia bor.: Jizerské Mts, Oldřichov v Hájích, Špičák (5156), 600 m, yellow pans, 17.5.-3.6.2003, 2ð, 3.-

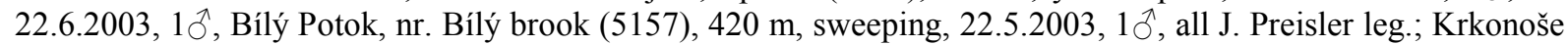
Mts, Horní Lánov, Pekelský brook (5360), $550 \mathrm{~m}$, Malaise trap, 5.2009, $1 \mathrm{O}^{\lambda}$, Svoboda nad Úpou, "Slunečná stráň” (5360), 645 m, Malaise trap, 2.-30.6.2009, 30ે, all M. Barták leg., all J. Starý det. et coll.

Distribution: Czech Republic (Moravia), Finland, Germany, Italy, Romania, Slovakia, Switzerland, Ukraine. First records from Bohemia.

\section{Tasiocera (Dasymolophilus) fuscescens (Lackschewitz, 1940)}

Bohemia bor.: Jizerské Mts, Jizerka, Bukovec (5158), 920 m, yellow pans, 9.-23.7.2003, 1ðิ , J. Preisler \& P. Vonička leg., J. Starý det. et coll.

Distribution: Widely distributed in Europe, including Czech Republic (Moravia) and Slovakia. First record from Bohemia.

\section{Tasiocera (Dasymolophilus) jenkinsoni Freeman, 1951}

Bohemia bor.: Krkonoše Mts, Horní Lánov, Pekelský brook (5360), 550 m, Malaise trap, 30.7.-18.8.2009, 2ð’, M. Barták leg., J. Starý det. et coll. 
Distribution: Czech Republic (Moravia), Germany, Great Britain, Hungary, Italy, Macedonia, Poland, Slovakia. First record from Bohemia.

\section{TIPULIDAE}

\section{Tipula (Lunatipula) magnicauda Strobl, 1895}

Moravia bor.: Štramberk, Kotouč, „Jurův kámen“ (6474), 21.5.2014, 10̂, A. Čelechovský leg., A. Čelechovský \& J. Starý det., A. Čelechovský coll.

Distribution: Austria, Belgium, Czech Republic (Bohemia), France, Germany, Italy, Poland, Spain, Switzerland. First record from Moravia. The record from Bohemia in all checklist versions (Martinovský 1987, 1997, Starý 2006, 2009) is based on the following, so far unpublished primary data: Bohemia centr., Český kras, Srbsko (6050), 28.6.1986, 1ठ, Z. Starý leg., Srbsko, Doutnáč Mt. (6050), 10.6.1993, 2ð̂, 3 q, J. Martinovský leg., all J. Martinovský det., in coll. Slezské zemské muzeum, Opava.

\section{Discussion}

Five species treated here as new to Bohemia are generally common and widely distributed in Europe, sometimes even outside it, and their absence in Bohemia up to now is only due to less intensive collecting activity in this part of the Czech Republic. Molophilus (M.) priapoides, M. (M.) variispinus, Ormosia (O.) moravica, Tasiocera (D.) exigua, T. (D.) jenkinsoni, and Tipula (L.) magnicauda are local, with scattered records throughout Europe. The latter species, a tipulid new to Moravia, probably represents the rarest and ecologically considerably significant addition to the fauna.

Acknowledgements: We thank M. Barták (Česká zemědělská univerzita, Praha), J. Preisler (Liberec), J. Vaněk (Vrchlabí), and P. Vonička (Severočeské muzeum, Liberec) for providing us with highly interesting material. The paper was financially supported by the Ministry of Culture of the Czech Republic by institutional financing of long-term conceptual development of the research institution (The Silesian Museum, MK000100595), internal grant of the Silesian Museum, No. IGS201505/2015.

\section{References}

Martinovský J. (1987): Tipulidae, pp. 11-14. In Ježek J. (ed.): Enumeratio insectorum Bohemoslovakiae Check list of Czechoslovak insects 2 (Diptera). Acta faunistica entomologica Musei Nationalis Pragae 18: 1341.

- (1997): Tipulidae, pp. 15-17. In Chvála M. (ed.): Check List of Diptera (Insecta) of the Czech and Slovak Republics. Karolinum - Charles University Press, Prague, 130 pp.

Oosterbroek P. (2015): Catalogue of the Craneflies of the World (Insecta, Diptera, Nematocera, Tipuloidea). Version 29 June 2015. Available from http://ccw.naturalis. nl

Starý J. (2006): Tipulidae Latreille, 1802. In Jedlička L., Stloukalová V. \& Kúdela M. (eds): Checklist of Diptera of the Czech Republic and Slovakia. Electronic version 1. Available from http://www.edvis.sk/ diptera2006/families/Tipulidae.htm.

- (2009): Tipulidae Latreille, 1802. In Jedlička L., Kúdela M. \& Stloukalová V. (eds): Checklist of Diptera of the Czech Republic and Slovakia. Electronic version 2. Available from http://www.edvis.sk/ diptera2009/families/tipulidae.htm.

Authors’ addresses: Jaroslav Starý, Neklanova 7, CZ-779 00 Olomouc-Nedvězí \& Silesian Museum, Nádražní okruh 31, CZ-746 01 Opava, Czech Republic; e-mail: stary.cranefly@gmail.com.

Alois Čelechovský, Department of Zoology and Laboratory of Ornithology, Palacký University, 17. listopadu 50, CZ-771 46, Olomouc, Czech Republic;

e-mail: celechov@prfnw.upol.cz. 